\title{
Willingness to Participate in At-Home HIV Testing Among Young Adults Who Use Opioids in Rural Appalachia
}

\author{
April M. Ballard ${ }^{1,2} \mathbb{D} \cdot$ Regine Haardöerfer $^{3} \cdot$ Nadya Prood $^{3} \cdot$ Chukwudi Mbagwu $^{4} \cdot$ Hannah L. F. Cooper $^{3}$. \\ April M. Young ${ }^{1,5}$
}

Published online: 10 September 2020

(c) Springer Science+Business Media, LLC, part of Springer Nature 2020

\begin{abstract}
New HIV infections associated with injection drug use are of major concern in rural US communities. This study explores acceptability of, consent for, and uptake of free at-home HIV testing among people who use drugs (PWUD) in one of the nation's epicenters for drug-related harms and HIV vulnerability: Rural Central Appalachia. Eligible participants were 18-35 years old, lived in Appalachian Kentucky, and reported using opioids to get high in the previous 30 days. A majority reported being likely $(63.6 \%, 96 / 151)$ to take a free at-home HIV tests and $66.9 \%(101 / 151)$ consented to receive one. Among those who were randomly selected to receive a Home Access HIV-1 test kit $(\mathrm{n}=37), 37.8 \%$ mailed in blood spots and $21.6 \%$ called to receive results. This study provides evidence that PWUD may be willing to take an at-home test, but other barriers may inhibit actual completion.
\end{abstract}

Keywords HIV $\cdot$ Home-based testing $\cdot$ Rural health $\cdot$ Substance-related disorders

\section{Introduction}

Rural communities such as those in central Appalachia have been disproportionately impacted by substance use and drug-related harms [1-5]. With the growing rate of injection drug use (IDU) and the emerging hepatitis $\mathrm{C}$ (HCV) epidemic in the United States (US) [6-11], new HIV infections in these communities are of major concern. While

April M. Ballard

april.ballard@emory.edu

1 Department of Epidemiology, University of Kentucky College of Public Health, Lexington, KY, USA

2 Gangarosa Department of Environmental Health, Emory University Rollins School of Public Health, 1518 Clifton Road NE, Atlanta, GA 30329, USA

3 Department of Behavioral, Social, and Health Education Sciences, Emory University Rollins School of Public Health, Atlanta, GA, USA

4 Department of Preventive Medicine and Environmental Health, University of Kentucky College of Public Health, Lexington, KY, USA

5 Center on Drug and Alcohol Research, Department of Behavioral Science, University of Kentucky College of Medicine, Lexington, KY, USA
Scott County, Indiana's HIV outbreak in 2014-2015 [11, 12] is most notable, clusters of new HIV infections associated with IDU continue to emerge in various states across the US including West Virginia [9, 13, 14] and Massachusetts [15]. Central to prevention efforts to mitigate the growing incidence of HIV transmission among people who inject drugs (PWID) are services that can provide testing and linkage to care. Despite having high rates of IDU [16], rural areas have limited HIV prevention and testing infrastructure [17, 18] and rural persons are less likely to report having ever been tested for HIV compared to their urban counterparts [18, 19] due to various known and unknown structural barriers.

Given limited HIV prevention and testing infrastructure $[17,18]$ and the roles that stigma, lack of confidentiality, and barriers to transportation play in suboptimal uptake of HIV testing and prevention services in rural communities [20], at-home HIV testing may present an innovative opportunity to increase access to and frequency of HIV testing among PWID [21-23]. Studies have explored acceptability and uptake of at-home HIV testing involving dried blood spots, but these have primarily been conducted among men who have sex with men (MSM) [21, 24-29]. One of the few studies to examine uptake of at-home HIV testing in a broader sample occurred more than 20 years ago, prior to FDA approval of the first at-home option in 1996 which was 
a dried blood spot test [24]. More current research among HIV-negative MSM in the US found that $62 \%$ were very likely and $20 \%$ were somewhat likely to self-report willingness to take a free at-home HIV test if it were offered as part of an online study. Willingness was higher among those who were offered a modest incentive ( $\$ 10$ or $\$ 25$ ), were black, had unprotected anal sex in the past 12 months, and were unaware of their HIV status [25]. Further, a longitudinal, randomized control trial conducted in 2013 among HIV-negative MSM in the US found that $79 \%$ of those who were sent an at-home HIV test kit requiring a finger stick returned their kit. Participants received $\$ 20$ for returning their at-home test kit and were randomized to receive online follow-up surveys or text message follow-up surveys. No significant difference was observed among randomization arm and return of the at-home test kit [26].

Existing research highlights the potential of at-home HIV tests to increase testing uptake among high-risk populations. However, acceptability of, consent for, and uptake of at-home HIV testing have not been investigated among rural people who use drugs (PWUD) to our knowledge. This pilot study aims to explore the acceptability and feasibility of free at-home blood spot HIV testing via the Home Access HIV-1 Test System among young, rural PWUD, most of whom inject drugs. This study was conducted in Central Appalachian Kentucky, a region that ranks among the highest in the nation for vulnerability to an HIV/HCV outbreak among PWID [30]. Additionally, four of the five counties in the study area are designated as medically underserved [31], underscoring the lack of medical resources available to those living in this area.

\section{Methods}

\section{Overview}

Participants were recruited from August 2017 to July 2018 for a web-based survey programmed in SurveyGizmo [32] using web-based peer referral and targeted outreach methods including hosting community cookouts to advertise the study, distributing flyers at community venues where young PWUD may be (e.g., social service organizations, laundromats, gas stations), walks through neighborhoods, and referrals from staff from a concurrent substance use study. Recruitment methods for the study are described in detail elsewhere [33]. Participants were deemed eligible if they were 18 to 35 years old, lived in one of five counties in rural Central Appalachian Kentucky, and reported using opioids to get high in the past 30 days. The study was funded to focus on individuals aged 18 to 35 years because of the disproportionate burden of opioid use and related harms (i.e., $\mathrm{HCV}$ and overdose) impacting young adults in rural settings
[7, 34, 35]. Opioids included prescription pain pills, heroin, buprenorphine, methadone, and synthetic opioids. To be eligible to participate, participants had to pass an online "quiz" to verify their substance use and residential status. More details about online eligibility verification are published elsewhere [36]. Informed consent was self-administered for both the online screening and survey, and included a brief consent quiz to ensure individuals comprehended the consent form content and were cognitively able to consent for participation.

The survey contained questions about participants' substance use, sexual and drug-related risk behaviors, and drug-related harms (e.g., overdose, history of HCV diagnosis). Lifetime IDU and recent (past 6 months) sexual and drug-related risk behaviors were queried; the latter included number of sex partners, frequency of condom use, IDU, and sharing of needles or syringes and other injection drug use equipment. It also included a detailed description of the at-home HIV testing procedure and a question that asked whether they consented to participate in at-home HIV testing. Specifically, participants were asked "Would you be willing to participate in at-home HIV testing? We will randomly select approximately 40 people to participant in athome HIV testing." Participants who consented were asked to provide a mailing address where the testing kit could be sent. SurveyGizmo was programmed to randomly select participants to be invited to complete an HIV test if they consented. Participants received $\$ 30$ for completing the survey and those selected for HIV testing received $\$ 25$ for completing at-home HIV testing as described in the 'At-Home HIV Testing' section below. Participants chose whether they wanted to receive incentives by mailed cash, money wire, mailed gift card, or an e-giftcard.

\section{Measures}

This study investigated three outcomes related to testing: (1) willingness to take an at-home HIV test; (2) consent to take an at-home HIV test if randomly selected; and (3) actual uptake of at-home HIV testing. Willingness to take an at-home HIV test was hypothetical and measured on a 4-point Likert scale. In the survey, participants were asked how likely they were to agree to take an at-home HIV test that would be sent in the mail in an unmarked box that required a person to prick their finger, send the blood spot to a company, and call to get their results and get linked to care if they have HIV. Participants could decline to answer the question, as well as respond 'don't know.' Participants who reported being somewhat or very unlikely to take an HIV test were prompted for reasons for disinterest via a select all question, including options related to uncertainty about test accuracy, wanting to talk to a counselor, and characteristics of the specific test kit (e.g., not wanting to provide a mailing 
address or to prick fingers for blood). Participants also had the option to write in other reasons. Consent to take an athome HIV test was captured through the informed consent process. Consenting to take an at-home HIV test meant participants were eligible to be randomly selected to receive a test kit. Uptake of at-home HIV testing was based on if participants who received their test kits sent their blood spot to Home Access, called for their results, and performed these tasks in the appropriate timeframe (i.e., within 3 weeks). Additional measures analyzed included sociodemographic items (e.g., gender, age, and education); method by which they were recruited (i.e., via staff contact, peer referral, or another method); and sexual and drug-related risk behavior items (see Analysis section and variables listed in Table 1).

\section{At-Home HIV Testing}

HIV test kits were purchased from Home Access Health Corporation prior to enrolling participants in the study and stored at a research office in Atlanta, Georgia. The first athome testing option, Home Access HIV-1 Test System, was approved by the FDA in 1996 and uses a finger-prick blood test. The Home Access System requires users to send their blood sample to a lab for testing then to call a toll-free number using an anonymous code to receive results and posttest counseling, which includes education and counseling about the HIV-1 antibody test, HIV prevention, risk reduction behavior, and AIDS. According to Home Access, if test results are positive, the counselor evaluates the coping skills, availability of social support, and person's ability to inform their sexual and needle-sharing partners. Counselors also provide referrals to local physicians and services that provide assistance with partner notification, as well as referrals to the National AIDS Hotline and other psychosocial services as necessary [37].

Throughout the study, study staff checked an online survey database daily to determine if any new participants had completed the survey and had been randomly selected to receive an HIV test kit. If a participant was chosen, the staff person registered the test kit with Home Access, recorded the test kit number and corresponding study ID number, and mailed the kit to the address the participant provided with postal tracking so that staff could ensure that it was delivered. Test kits were always sent within three days of

Table 1 Descriptive and bivariate analyses of participant demographic and behavioral characteristics and consent to take an at-home HIV test, drawn from a sample of people who use drugs $(\mathrm{N}=151)$ and live in rural Kentucky

\begin{tabular}{|c|c|c|c|c|}
\hline Participant characteristic & $\begin{array}{l}\text { Total }(\mathrm{n}=151) \\
\mathrm{n}(\%)\end{array}$ & $\begin{array}{l}\text { Consented to HIV test } \\
(\mathrm{n}=101) \\
\mathrm{n}(\%)\end{array}$ & $\begin{array}{l}\text { Did not consent to HIV } \\
\text { test }(\mathrm{n}=50) \\
\mathrm{n}(\%)\end{array}$ & P-value \\
\hline Age mean (standard deviation) & $28.9(4.1)$ & $29.3(3.9)$ & $28.0(4.4)$ & 0.06 \\
\hline \multicolumn{5}{|l|}{ Gender } \\
\hline Male & $93(61.6)$ & $60(59.4)$ & $33(66.0)$ & \\
\hline Female & $58(38.4)$ & $41(40.6)$ & $17(34.0)$ & 0.43 \\
\hline \multicolumn{5}{|l|}{ Education $(\mathrm{n}=148)$} \\
\hline Less than 12 th grade & $46(31.1)$ & $23(23.5)$ & $23(46.0)$ & \\
\hline Completed high school or GED & $102(68.9)$ & $75(76.5)$ & $27(54.0)$ & $<0.01$ \\
\hline Homeless $(\mathrm{n}=149)$ (past 6 months) & $68(45.6)$ & $43(43.4)$ & $25(50.0)$ & 0.45 \\
\hline Transportation problems $(\mathrm{n}=150)$ (past 6 months) & $107(71.3)$ & $74(73.3)$ & $33(67.4)$ & 0.45 \\
\hline \multicolumn{5}{|l|}{ Recruitment type } \\
\hline Peer referral & $42(27.8)$ & $17(16.8)$ & $25(50.0)$ & \\
\hline Targeted outreach with staff contact & $100(66.2)$ & $77(76.2)$ & $23(46.0)$ & \\
\hline Other & $9(6.0)$ & $7(6.9)$ & $2(4.0)$ & $<0.01$ \\
\hline Ever had HIV test $(\mathrm{n}=147)$ & $93(63.3)$ & $69(68.3)$ & $24(52.2)$ & 0.06 \\
\hline Inconsistent condom use $(\mathrm{n}=140)^{\mathrm{a}}$ & $119(85.0)$ & $87(92.6)$ & $32(69.6)$ & $<0.01$ \\
\hline More than 1 sex partner (past 6 months) & $67(44.4)$ & $43(42.6)$ & $24(48.0)$ & 0.53 \\
\hline Lifetime history of IDU $(n=145)$ & $115(79.3)$ & $83(83.8)$ & $32(69.6)$ & 0.05 \\
\hline Injected drugs $(\mathrm{n}=145)$ (past 6 months) & $100(69.0)$ & $75(75.8)$ & $25(54.4)$ & 0.01 \\
\hline Shared syringes or needles $(n=141)$ (past 6 months) & $55(39.0)$ & $45(45.9)$ & $10(23.3)$ & 0.01 \\
\hline Shared drug use equipment $(\mathrm{n}=139)^{\mathrm{b}}$ (past 6 months) & $61(43.9)$ & $50(51.6)$ & $11(26.2)$ & $<0.01$ \\
\hline
\end{tabular}

GED general education development

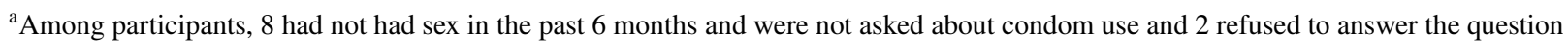

${ }^{\mathrm{b}}$ Two participants refused to answer 
random selection and the exterior of the package did not contain any information about the contents. To receive their \$25 incentive for completing HIV testing, participants were required to (1) send in their specimen within 10 days of sample collection, a period based on blood sample expiration, and (2) call Home Access to receive their results within a 3-week period. A staff member contacted a designated representative at Home Access to determine if participants sent their specimen to Home Access and the date they called to retrieve their results; we did not receive individual test results from Home Access due to the sensitivity of the data, the bias it could introduce in individuals' reported willingness to participate in at-home testing (our intent to collect test result data would need to be disclosed to them in the consent form preceding the survey), and the lack of statistical power we would have to use test results as an outcome in multivariable models.

The test consisted of a finger-prick blood test with a sensitivity and specificity greater than $99.9 \%$ [37]; and the kit also contained a pamphlet with pre-test counseling information, and information on how to perform the test and call for results. Study staff also included a letter in each kit that reiterated the compensation protocol and included Home Access and study contact information. The Home Access system was selected based on the high sensitivity and specificity of the blood spot test, availability of pre- and post-test counseling, and the ability to track whether participants had (1) mailed in the blood spot and (2) called to receive results.

\section{Analyses}

Descriptive statistics were utilized to describe willingness to take an at-home HIV test, reasons for being unwilling to take a test, and uptake of at-home testing. Bivariate and logistic regression analyses were conducted to examine demographic and behavioral factors associated with consent to take a free at-home HIV test among rural, young adult PWUD in Appalachian Kentucky. Specifically, five separate multivariable logistic regression models, adjusting for demographic variables, were run to investigate the relationship between the following independent variables of interest and consent to take an at-home HIV test: (1) ever having an HIV test; (2) inconsistent condom use in the past 6 months; (3) having more than one sex partner in the past 6 months; (4) IDU in the past 6 months; and (5) sharing syringes or needles in the past 6 months. Demographic variables that were associated with the outcome in bivariate analyses with $p<0.10$ were included as covariates in multivariable models (i.e., age, education, and recruitment type). Two-way interaction between each possible pair of variables was examined and no significant interaction was detected. Multi-collinearity among variables in each model was examined and was not present for any of the models. Analyses were performed using SAS 9.4.

Of note, the relationship between sharing drug use equipment in the past 6 months and consent to take an at-home HIV test was not explored via multivariable logistic regression models as most participants who reported sharing syringes or needles (92.6\%) also reported sharing other drug use equipment, and most that reported not sharing syringes (87.0\%) also reported not sharing other drug use equipment. Similarly, the relationship between lifetime IDU and consent to take an at-home test was not explored because most participants who had a history of IDU (87.0\%) reported IDU in the past 6 months, and all participants who reported no lifetime IDU reported no IDU in the past 6 months.

\section{Ethics}

The Emory University Institutional Review Board approved all study procedures and data were protected by a Federal Certificate of Confidentiality

\section{Results}

\section{Overview}

Overall, 410 online eligibility screeners were completed, and 234 participants were deemed eligible, consented to participate, and completed the survey. To maximize data quality and validity of survey entries, several methods were leveraged to detect and eliminate fraud including components of a fraud-detection protocol created by Ballard and colleagues to identify fraud in web-based surveys [38]. Eighty-three survey entries were deemed fraudulent and were excluded from analyses. The final sample thus included 151 valid surveys. More details on fraud detection can be found elsewhere [36, 39].

Participants were 28.9 years old on average (standard deviation $[\mathrm{SD}]=4.1)$ and $61.6 \%$ were men. Most participants identified as White (96.7\%) and non-Hispanic (98.7\%). Overall, participants reported having a mean monthly income of \$300 (SD: \$664) and 45.0\% reported experiencing homelessness (i.e., living on the street, or in a car, park, abandoned building, or shelter) in the past 6 months. Heroin (72.2\%) was the most frequently reported opioid used to get high in the past 6 months, and 51.7\% reported using prescription pain pills. Two-thirds of the sample self-reported injecting drugs in the past 6 months.

\section{Willingness and Consent to Participate in At-Home HIV Testing}

Of the 151 survey participants, $63.6 \%$ were somewhat or very likely to hypothetically take (i.e., were willing to 
take) an at-home HIV test and $11.9 \%$ were somewhat or very unlikely. Thirty participants did not know how likely they were to take an at-home HIV test and five refused to answer. A majority of participants $(66.9 \%)$ consented to take at-home HIV testing if they were randomly selected.

Table 1 presents results of bivariate analyses by whether participants did or did not consent to take an at-home HIV test. Overall, a greater proportion of those who consented to take part in HIV testing had completed high school or a GED compared to those who did not consent $(p<0.01)$. Those who consented to take at-home HIV tests were also more likely to be recruited by study staff ( $p<0.01$ ), to inconsistently use condoms (i.e., did not use a condom every time they had sex $)(p<0.01)$, to have a lifetime history of IDU $(p=0.05)$, to report IDU in the past 6 months $(p=0.01)$, to share syringes or needles in the past 6 months $(p=0.01)$, and to share other drug equipment in the past 6 months ( $\mathrm{p}<0.01)$. There were no significant associations between consent to take an HIV test and being tested for HIV previously $(p=0.06)$, age $(p=0.06)$, gender $(p=0.43)$, homeless status $(\mathrm{p}=0.45)$, transportation problems (i.e., being unable to do something that they needed to because they did not have a way to get there) $(\mathrm{p}=0.45)$, and having multiple sex partners $(\mathrm{p}=0.53)$.

Among those who were somewhat or very unlikely to take an at-home HIV test $(\mathrm{n}=18)$, being uncertain about the test's accuracy $(22.2 \%)$ and being tested very recently $(22.2 \%)$ were the top reasons for why they were unwilling to take the test. Participants also reported that they would rather talk to a counselor $(n=3)$, they did not think they needed an HIV test $(n=3)$, they did not want to give their mailing address $(n=1)$, and they did not want to stick their finger for blood $(n=1)$.

Table 2 presents the results from multivariable logistic regression analyses to examine the associations between sexual and drug-related risk behaviors and consent to take an at-home HIV test controlling for age, education, and recruitment type. While controlling for age, education, and recruitment type, inconsistent condom use and syringe/needle sharing in the past 6 months were statistically significantly associated with a participant consenting to take an at-home HIV test. Specifically, those who used condoms inconsistently during sex and/or who shared syringes/needles were 3.82 (95\% confidence interval [CI] [1.29, 11.34], $\mathrm{p}=0.02)$

Table 2 Correlates associated with consent to take an at-home HIV test adjusting for age, education, and recruitment method

\begin{tabular}{|c|c|c|c|c|c|}
\hline & $\begin{array}{l}\text { Model 1a AOR (95\% } \\
\text { CI) }\end{array}$ & $\begin{array}{l}\text { Model 2 } 2^{\mathrm{b}} \text { AOR (95\% } \\
\text { CI) }\end{array}$ & $\begin{array}{l}\text { Model 3c AOR (95\% } \\
\text { CI) }\end{array}$ & $\begin{array}{l}\text { Model } 4^{\mathrm{d}} \text { AOR (95\% } \\
\text { CI) }\end{array}$ & $\begin{array}{l}\text { Model } 5^{\mathrm{e}} \\
\text { AOR }(95 \% \mathrm{CI})\end{array}$ \\
\hline Ever had HIV test & $1.00(0.98,1.01)$ & & & & \\
\hline $\begin{array}{l}\text { Inconsistent condom } \\
\text { use (past } 6 \text { months) }\end{array}$ & & $3.82(1.29,11.34)^{*}$ & & & \\
\hline $\begin{array}{l}\text { More than } 1 \text { sex part- } \\
\text { ner (past } 6 \text { months) }\end{array}$ & & & $1.30(0.58,2.89)$ & & \\
\hline $\begin{array}{l}\text { Injected drugs (past } \\
6 \text { months) }\end{array}$ & & & & $2.15(0.95,4.86)$ & \\
\hline $\begin{array}{l}\text { Shared syringes or nee- } \\
\text { dles (past } 6 \text { months) }\end{array}$ & & & & & $2.38(1.00,5.72)^{*}$ \\
\hline Age & $1.05(0.96,1.15)$ & $1.02(0.93,1.13)$ & $1.06(0.97,1.16)$ & $1.05(0.96,1.15)$ & $1.03(0.93,1.13)$ \\
\hline \multicolumn{6}{|l|}{ Education } \\
\hline Less than 12 th grade & Ref & Ref & Ref & Ref & Ref \\
\hline $\begin{array}{l}\text { Completed high } \\
\text { school or GED }\end{array}$ & $2.41(1.08,5.35)^{*}$ & $2.69(1.14,6.33)^{*}$ & $2.19(1.00,4.83)^{*}$ & $2.70(1.19,6.15)^{*}$ & $2.94(1.27,6.80)^{*}$ \\
\hline \multicolumn{6}{|l|}{ Recruitment type } \\
\hline Peer referral & Ref & Ref & Ref & Ref & Ref \\
\hline $\begin{array}{l}\text { Targeted outreach } \\
\text { with staff contact }\end{array}$ & $3.46(1.52,7.83)^{*}$ & $3.84(1.64,8.99)^{*}$ & $4.62(1.99,10.77)^{*}$ & $2.83(1.21,6.64)^{*}$ & $3.57(1.51,8.44)^{*}$ \\
\hline Other & $2.80(0.49,16.14)$ & $2.32(0.40,13.42)$ & $3.82(0.66,22.26)$ & $2.01(0.33,12.44)$ & $1.54(0.24,9.76)$ \\
\hline
\end{tabular}

$A O R$ adjusted odds ratio, $C I$ confidence interval, GED general education development

${ }^{a}$ Model includes ever having an HIV test, age, education, and recruitment type

${ }^{\mathrm{b}}$ Model includes inconsistent condom use in the past 6 months, age, education, and recruitment type

${ }^{c}$ Model includes having more than one sex partner in the past 6 months, age, education, and recruitment type

${ }^{\mathrm{d}}$ Model includes IDU in the past 6 months, age, education, and recruitment type

${ }^{\mathrm{e}}$ Model includes sharing syringes or needles in the past 6 months, age, education, and recruitment type

* $\mathrm{p}<0.05$ 
and $2.38(95 \% \mathrm{CI}[1.00,5.72], \mathrm{p}=0.05)$ times more likely to consent to take an at-home HIV test, respectively, compared to their counterparts. Associations between IDU in the past 6 months (adjusted odds ratio $[\mathrm{AOR}]=2.15,95 \%$ CI [0.95, 4.86], $\mathrm{p}=0.07)$, having more than one sex partner in the past 6 months $(\mathrm{AOR}=1.30,95 \% \mathrm{CI}[0.58,2.89], \mathrm{p}=0.52)$, and previously being tested for HIV (AOR $=1.00,95 \% \mathrm{CI}$ $[0.98,1.01], \mathrm{p}=0.89)$ and consenting to take an at-home HIV test did not reach statistical significance.

Across all five separate logistic regression models, education and recruitment type were significantly associated with consent to take an at-home HIV test. Specifically, those who had completed high school or a GED were more than 2.19-2.94 times more likely to consent to HIV testing compared to those who had not completed high school. Additionally, those who were recruited via staff outreach and other methods were $2.83-4.62$ and $1.54-3.82$ times more likely to consent to take an HIV test compared to those who were recruited through peer referral across models.

\section{Uptake of At-Home HIV Testing}

Figure 1 displays data on survey participants and uptake of at-home HIV testing. Thirty-eight randomly selected participants who consented to take an at-home test were sent Home Access HIV test kits via mail. However, one test kit was undeliverable to the participant due to change of address and inability to identify a new address. Among the 37 participants who received an at-home HIV test, $37.8 \%$ sent their test kits with the completed blood spot to Home Access, among whom $64.3 \%$ (9/14) called Home Access for their results. Of those who called for their results, $88.9 \%(8 / 9)$ called within the 3 -week eligibility period. Thus, $21.6 \%$ (8/37) of participants who received at-home HIV test kits completed the testing process successfully.

\section{Discussion}

Innovative methods of expanding HIV testing in rural communities are needed as rates of IDU continue to increase and as lack of HIV prevention and testing infrastructure, stigma, structural barriers, and perceived lack of confidentiality contribute to suboptimal uptake of traditional HIV testing. Athome testing services may present an opportunity to increase access and frequency of testing, however willingness, consent for, and uptake have not been investigated among rural PWUD. This exploratory study investigated the acceptability and feasibility of free at-home HIV testing among young, rural PWUD in central Appalachia, most of whom inject. The study revealed that over half of participants were somewhat or very willing to take an at-home test $(63.6 \%)$ and more than two-thirds consented to take an at-home HIV test
(66.9\%), but only $21.6 \%$ of those who received an HIV test completed the process successfully. As such, this study provides evidence that young, rural PWUD may be willing to take an at-home test, but other barriers may inhibit actual completion. Results also suggest that those who engaged in sexual and drug-related risk behaviors may be more likely to accept an HIV test, and recruitment methods involving faceto-face staff contact were associated with increased consent to at-home HIV testing.

The proportion of rural PWUD in this study who were willing to take an at-home HIV test was similar to or greater than what has been shown in previous research among other populations, including MSM recruited using webbased methods [25, 40] and the general population [24]. In contrast, previous studies investigating actual uptake of blood-based HIV self-testing in high-income countries have found test completion ranging from 77.9 to $90.0 \%$ [28, 41, 42], which is substantially higher than among rural PWUD in this study (21.6\%). However, direct comparison of findings is difficult given methodological differences between studies. For example, previous studies have involved staff instructing and observing participants' self-administered testing [28, 41, 42], had larger sample sizes [28, 41, 42], and recruited participants at HIV and sexually transmitted infection (STI) testing programs or facilities [28, 41]. These studies also did not focus on or include PWUD and were not conducted exclusively in rural areas where HIV stigma may be heightened [28, 41, 42].

In this sample of rural PWUD, those who used condoms inconsistently and shared syringes/needles were more likely to consent to take an at-home HIV test. Engagement in other risk behavior was not significantly associated with consent to participate in at-home HIV testing indicating that those who engaged in other drug- and sex-related risk behaviors (i.e., IDU and multiple sex partners) in the past 6 months were no less likely to consent to take an at-home test than their counterparts. While small sample size limited statistical power, these exploratory findings are encouraging: PWUD at highest risk for HIV (i.e., those who inconsistently use condoms and/or shared syringes/needles) appear to be more likely to be willing to participate in at-home HIV testing than their counterparts.

Of note, those who were recruited into the study through targeted outreach with staff were more likely to consent to take an at-home HIV test. Rapport may therefore be important among vulnerable populations such as rural PWUD, especially when piloting or introducing new approaches for sensitive topics such as HIV testing. The use of a local field office and context-specific, street-outreach activities to recruit rural PWUD provided opportunities for in-person contact between study staff and participants [33] that may be critical to maximizing at-home test acceptance. Anecdotally, participants revealed that personal contact with study 


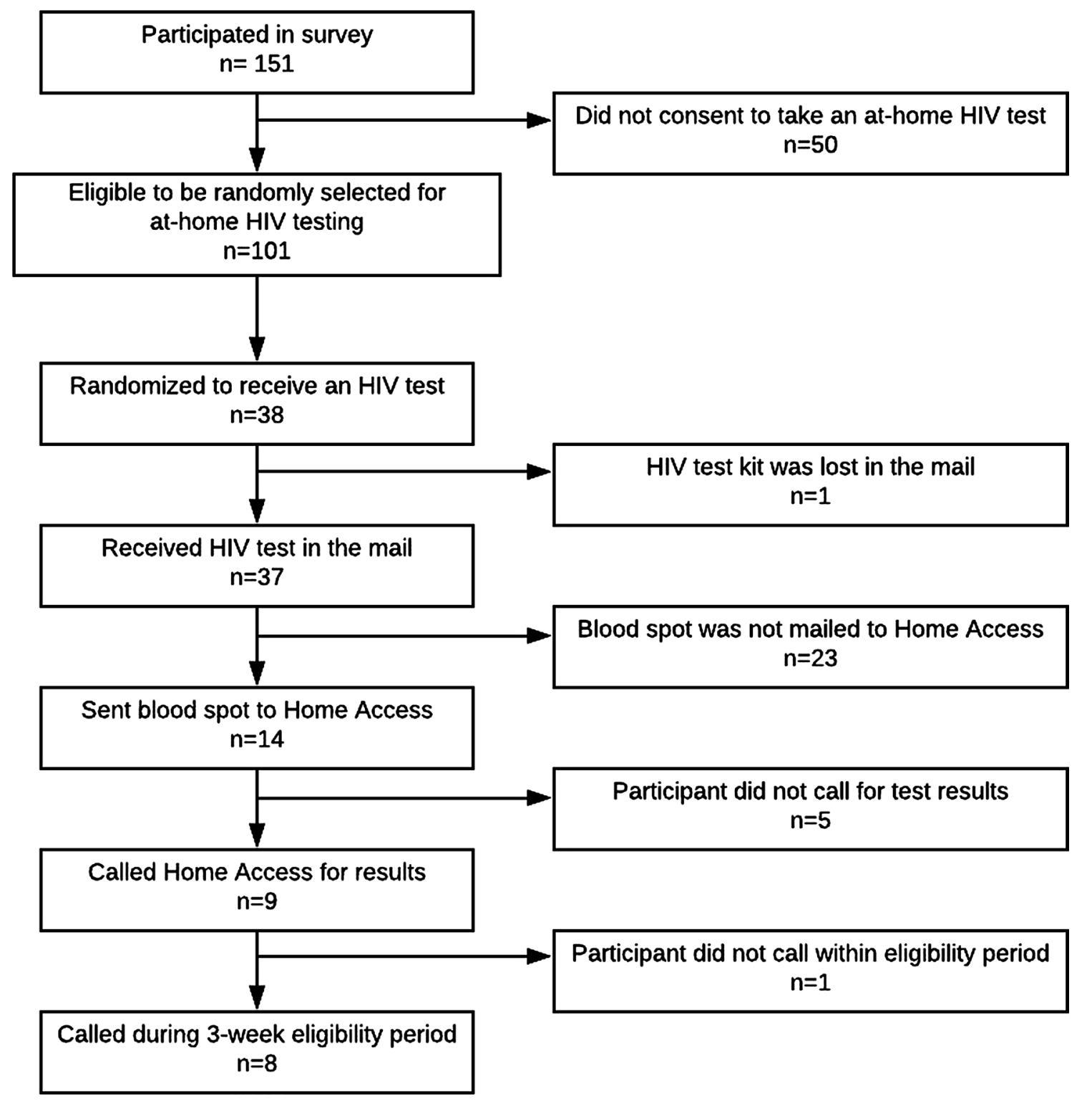

Fig. 1 Flow chart of survey participants and uptake of at-home HIV testing

staff increased rapport, trust, and perceived study legitimacy, though follow-up qualitative research is needed to fully understand the relationship between consent, test completion, and recruitment type.

A majority $(62.2 \%)$ of participants randomly selected to receive the test did not mail their completed tests to Home Access, and about one-third of participants who mailed their completed tests did not call to receive their results. Participants informally told study staff that the postal mail component of testing was difficult to navigate. For example, some participants had to take completed tests to a post office to send to Home Access because it would not fit in a household mailbox. Others reported simply forgetting to mail the completed dried blood spot. Mailing test kits and calling to get results may therefore be an added barrier that remains unexplored. Participants who reported being somewhat or very unlikely to take mailed at-home blood spot HIV tests were also queried about uncertainty within the survey instrument. Reasons reported by participants included being uncertain about the test's accuracy, being tested very recently, desire to talk to a counselor post-testing, thinking they did not need an HIV test, not wanting to give their mailing address, and not wanting to stick their finger for blood. Additional research is therefore needed to understand these and other unknown barriers. 


\section{Limitations}

A strength of this study was that it was able to examine willingness, consent to, and actual completion of at-home tests. However, the outcome for analyses was limited to consent because the number of participants in this pilot study who were randomly selected to receive an at-home HIV test kit was small, thereby limiting statistical power to investigate demographic and behavioral characteristics that may be related to actual uptake. Due to the crosssectional nature of this pilot study, we did not follow-up with participants to determine their test results and we also did not seek test results from Home Access. The latter was not pursued due to concern about biasing results. Specifically, if test results were to be pursued by our team, participants would have needed to be made aware of this plan and the down-stream consequences (i.e., reporting the case to the state health department) in the consent document; this research protocol could have swayed individuals' willingness to participate in at-home HIV testing making our outcome measure less reflective of real-world, at-home HIV test uptake. Because the research team did not have access to test results, Home Access was relied upon for post-test counseling and linkage to care among those (if any) who called and received a positive result, as is done in standard at-home HIV testing.

The web-based methods used for this study, while innovative and potentially more scalable than staff-intensive approaches to disseminate at-home HIV tests, also presented challenges and limitations. There was no option for participants to receive assistance or complete HIV test kits with study staff, which could have increased rapport, helped participants feel more comfortable, and provided a private space for specimen collection completion. Additionally, while the combined use of targeted outreach and web-based peer referral strengthened the sampling approach, the web-based nature of the study may limit the generalizability of these findings to individuals with less access to or comfort with web-based technologies.

Finally, the use of finger-prick HIV test kits and the Home Access system may be a limitation of this study, as it requires increased capability and effort on behalf of participants related to specimen collection via blood spots and mailing test kits as compared to at-home oral swab rapid kits. For example, almost half $(45.0 \%)$ of participants reported experiencing homelessness in the past 6 months, which could lead to decreased willingness and/ or ability to complete an at-home HIV test due to lack of residence (i.e., no address to send the kit to), a transient lifestyle (i.e., inability to carry and store the kit prior to completion), and/or lack of privacy (i.e., inability to privately complete the test). Acceptability and willingness to consent to take at-home HIV testing may have been higher with OraQuick's oral fluid HIV test given that it provides results in just 20 min and is less invasive [40, 43]. However, while oral swab rapid self-testing kits are suitable for some populations and settings as they do not require samples to be sent to a laboratory for analysis [23, 44], lower sensitivity in comparison to blood specimen and lack of counseling and supervision may lead to false reassurance and the possibility of risk compensation [23, 45].

Of note, sales of the Home Access HIV-1 Test System used in this study were discontinued effective January 1, 2019 [46] shortly following the completion of data collection. Nevertheless, sales of other blood (e.g., Everlywell, which is not FDA-approved) and oral at-home HIV tests (i.e., OraQuick, which is the sole at-home FDA-approved HIV test available in the US [47]) continue, and HIV selftesting technologies remain a potentially promising strategy to improve testing uptake among high-risk populations [48, 49]. COVID-19 and the associated control measures implemented in response have also resulted in renewed interest in at-home testing technologies [50], which might be particularly advantageous for populations who have difficulty accessing medical care.

\section{Conclusion}

At-home HIV testing presents an innovative opportunity to increase access to and frequency of HIV testing among at-risk populations such as rural PWUD. With increasing IDU in rural areas and emerging HIV cases [6-9, 12-15], approaches to better reach the needs of this population are warranted. This study revealed that a majority of rural PWUD may be willing to complete an at-home blood-spot HIV test, but unknown barriers may inhibit completion of tests or retrieval of results. Having to mail test kits and call to retrieve results may impede completion, requiring other inventive approaches to deliver at-home test kits and results to participants. Additionally, in-person contact via community-based staff may also be necessary to build rapport and facilitate willingness and uptake of tests.

Acknowledgements This study was funded by the National Institute on Drug Abuse (R21 DA042727; principal investigators (PIs): Cooper and Young). Community partners who provided feedback during the development of the survey were identified through an ongoing study supported by the National Institute on Drug Abuse, Centers for Disease Control and Prevention (CDC), Substance Abuse and Mental Health Services Administration (SAMHSA), and the Appalachian Regional Commission (UG3 DA044798; PIs: Young and Cooper); the content is solely the responsibility of the authors and does not necessarily represent the official views of the National Institutes of Health, CDC, SAMHSA, or ARC. The authors thank community researchers, Mary Beth Lawson, Travis Green, and Cindy Jolly for assistance with survey administration and logistics, Kevin Tillman for literature 
review assistance, as well as the Emory Center for AIDS Research (P30 AI050409; PIs: del Rio, Curran, Hunter), Nicole Luisi, and Danielle Lambert for technical support with survey programming.

\section{Compliance with Ethical Standards}

Conflict of interest The authors declare that they have no conflict of interest.

Ethical Approval The study was conducted in accordance with the ethical standards governing protection of human subjects and with the 1964 Helsinki Declaration and its later amendments or comparable ethical standards. Emory University's Institutional Review Board reviewed and approved this study.

Informed Consent Informed consent was obtained from all individual participants in the study.

\section{References}

1. Moody L, Satterwhite E, Bickel WK. Substance use in rural central appalachia: current status and treatment considerations. Rural Ment Health. 2017;41(2):123-35.

2. Center for Beavioral Health Statistics and Quality. Behavioral health trends in the United States: results from the 2014 National Survey on Drug Use and Health [Internet]. Bethesda, MD: HHS publication no. SMA 15-4927, NSDUH series H-50, 2015. https ://www.samhsa.gov/data/.

3. Paulozzi LJ, Xi Y. Recent changes in drug poisoning mortality in the United States by urban-rural status and by drug type. Pharmacoepidemiol Drug Saf. 2008;17(10):997-1005.

4. Young AM, Havens JR, Leukefeld CG. A comparison of rural and urban nonmedical prescription opioid users' lifetime and recent drug use. Am J Drug Alcohol Abuse. 2012;38(3):220-7.

5. Havens JR, Walker R, Leukefeld CG. Prevalence of opioid analgesic injection among rural nonmedical opioid analgesic users. Drug Alcohol Depend. 2007;87(1):98-102.

6. Des Jarlais DC, Nugent A, Solberg A, Feelemyer J, Mermin J, Holtzman D. Syringe service programs for persons who inject drugs in urban, suburban, and rural areas-United States, 2013. MMWR Morb Mortal Wkly Rep. 2015;64(48):1337-411.

7. Zibbell JE, Iqbal K, Patel RC, Suryaprasad A, Sanders KJ, MooreMoravian $\mathrm{L}$, et al. Increases in hepatitis $\mathrm{C}$ virus infection related to injection drug use among persons aged $\leq 30$ years-Kentucky, Tennessee, Virginia, and West Virginia, 2006-2012. MMWR Morb Mortal Wkly Rep. 2015;64(17):453-8.

8. Zibbell JE, Asher AK, Patel RC, Kupronis B, Iqbal K, Ward JW, et al. Increases in acute hepatitis $\mathrm{C}$ virus infection related to a growing opioid epidemic and associated injection drug use, United States, 2004 to 2014. Am J Public Health. 2018;108(2):175-81.

9. Donroe JH, Socias ME, Marshall BDL. The deepening opioid crisis in North America: historical context and current solutions. Curr Addict Rep. 2018;5(4):454-63.

10. Saloner B, McGinty EE, Beletsky L, Bluthenthal R, Beyrer C, Botticelli M, et al. A public health strategy for the opioid crisis. Public Health Rep. 2018;133(1_suppl):24S-34S

11. Beyrer C. No more Scott counties. Lancet HIV. 2018;5(10):e541-e542542.

12. Peters PJ, Pontones P, Hoover KW, Patel MR, Galang RR, Shields $\mathrm{J}$, et al. HIV infection linked to injection use of oxymorphone in Indiana, 2014-2015. N Engl J Med. 2016;375(3):229-39.
13. Evans ME, Labuda SM, Hogan V, Agnew-Brune C, Armstrong J, Periasamy Karuppiah AB, et al. Notes from the field: HIV infection investigation in a rural area-West Virginia, 2017. MMWR Morb Mortal Wkly Rep. 2018;67(8):257-8.

14. Bradley H, Hogan V, Agnew-Brune C, Armstrong J, Broussard D, Buchacz K, et al. Increased HIV diagnoses in West Virginia counties highly vulnerable to rapid HIV dissemination through injection drug use: a cautionary tale. Ann Epidemiol. 2019;34:12-7.

15. Cranston K, Alpren C, John B, Dawson E, Roosevelt K, Burrage A, et al. Notes from the field: HIV diagnoses among persons who inject drugs-Northeastern Massachusetts, 2015-2018. MMWR Morb Mortal Wkly Rep. 2019;68(10):253-4.

16. Keyes KM, Cerdá M, Brady JE, Havens JR, Galea S. Understanding the rural-urban differences in nonmedical prescription opioid use and abuse in the United States. Am J Public Health. 2014;104(2):e52-e5959.

17. Sutton M, Anthony M-N, Vila C, McLellan-Lemal E, Weidle PJ. HIV testing and HIV/AIDS treatment services in rural counties in 10 Southern States: service provider perspectives. J Rural Health. 2010;26(3):240-7.

18. Schafer KR, Albrecht H, Dillingham R, Hogg RS, Jaworsky $\mathrm{D}$, Kasper K, et al. The continuum of HIV care in rural communities in the United States and Canada: what is known and future research directions. J Acquir Immune Defic Syndr. 2017;75(1):35-44.

19. Ohl ME, Perencevich E. Frequency of human immunodeficiency virus (HIV) testing in urban vs. rural areas of the United States: results from a nationally-representative sample. BMC Public Health. 2011;11:681.

20. Pellowski JA. Barriers to care for rural people living with HIV: a review of domestic research and health care models. J Assoc Nurses AIDS Care. 2013;24(5):422-37.

21. Pant Pai N, Sharma J, Shivkumar S, Pillay S, Vadnais C, Joseph $\mathrm{L}$, et al. Supervised and unsupervised self-testing for HIV in high- and low-risk populations: a systematic review. PLoS Med. 2013;10(4):e1001414.

22. Figueroa C, Johnson C, Ford N, Sands A, Dalal S, Meurant R, et al. Reliability of HIV rapid diagnostic tests for self-testing compared with testing by health-care workers: a systematic review and meta-analysis. Lancet HIV. 2018;5(6):e277-e290290.

23. Steehler K, Siegler AJ. Bringing HIV self-testing to scale in the United States: a review of challenges, potential solutions, and future opportunities. J Clin Microbiol. 2019;57(11):e00257-e319.

24. Phillips KA, Flatt SJ, Morrison KR, Coates TJ. Potential use of home HIV testing. N Engl J Med. 1995;332(19):1308-11.

25. Sharma A, Sullivan PS, Khosropour CM. Willingness to take a free home HIV test and associated factors among internet-using men who have sex with men. J Int Assoc Physicians AIDS Care (Chic). 2011;10(6):357-64.

26. Khosropour CM, Johnson BA, Ricca AV, Sullivan PS. Enhancing retention of an Internet-based cohort study of men who have sex with men (MSM) via text messaging: randomized controlled trial. J Med Internet Res. 2013;15(8):e194.

27. de la Fuente L, Rosales-Statkus ME, Hoyos J, Pulido J, Santos $\mathrm{S}$, Bravo MJ, et al. Are participants in a street-based HIV testing program able to perform their own rapid test and interpret the results? PLoS ONE. 2012;7(10):e46555.

28. Lee VJ, Tan SC, Earnest A, Seong PS, Tan HH, Leo YS. User acceptability and feasibility of self-testing with HIV rapid tests. J Acquir Immune Defic Syndr. 2007;45(4):449-53.

29. Ricca AV, Hall EW, Khosropour CM, Sullivan PS. Factors associated with returning at-home specimen collection kits for HIV testing among Internet-using men who have sex with men. J Int Assoc Prov AIDS Care. 2016;15(6):463-9.

30. Van Handel MM, Rose CE, Hallisey EJ, Kolling JL, Zibbell JE, Lewis B, et al. County-level vulnerability assessment for rapid 
dissemination of HIV or HCV infections among persons who inject drugs, United States. JAIDS J Acquir Immune Defic Syndr. 2016;73(3):323-31.

31. Medically Underserved Areas Find [Internet]. Maryland: Health Resources \& Services Administration. https://data.hrsa.gov/tools /shortage-area/mua-find.

32. Bharel M, Casey C, Wittenberg E. Disparities in cancer screening: acceptance of pap smears among homeless women. J Womens Health. 2009;18(12):2011-6.

33. Young A, Ballard A, Cooper H. Novel recruitment methods for research among rural young adults who use opioids: cookouts, coupons, and community-based staff. Public Health Rep 2020 (in press).

34. Valdiserri R, Khalsa J, Dan C, Holmberg S, Zibbell J, Holtzman $\mathrm{D}$, et al. Confronting the emerging epidemic of $\mathrm{HCV}$ infection among young injection drug users. Am J Public Health. 2014;104(5):816-21.

35. Zibbell JE, Hart-Malloy R, Barry J, Fan L, Flanigan C. Risk factors for $\mathrm{HCV}$ infection among young adults in rural New York who inject prescription opioid analgesics. Am J Public Health. 2014;104(11):2226-32.

36. Ballard AM, Cooper HLF, Young AM. Web-based eligibility quizzes to verify opioid use and county residence among rural young adults: eligibility screening results from a feasibility study. JMIR Res Protoc. 2019;8(6):e12984.

37. Home Access Health Corporation. Home Access HIV-1 test system summary of safety and effectiveness [Internet]. Maryland: Premarket Approvals from the U.S. Food \& Drug Administration; 1994. https://www.wayback.archive-it.org/7993/2017011114 4040/https://www.fda.gov/BiologicsBloodVaccines/BloodBlood Products/ApprovedProducts/PremarketApprovalsPMAs/ucm09 1475.htm.

38. Ballard AM, Cardwell T, Young AM. Fraud detection protocol for web-based research among men who have sex with men: development and descriptive evaluation. JMIR Public Health Surveill. 2019;5(1):e12344.

39. Cooper HLF, Crawford ND, Haardörfer R, Prood N, Jones-Harrell $\mathrm{C}$, Ibragimov $\mathrm{U}$, et al. Using web-based pin-drop maps to capture activity spaces among young adults who use drugs in rural areas: cross-sectional survey. JMIR Public Health Surveill. 2019;5(4):e13593.

40. Sharma A, Stephenson RB, White D, Sullivan PS. Acceptability and intended usage preferences for six HIV testing options among internet-using men who have sex with men. SpringerPlus. 2014;3(1): 109 .

41. Belza MJ, Rosales-Statkus ME, Hoyos J, Segura P, Ferreras E, Sánchez R, et al. Supervised blood-based self-sample collection and rapid test performance: a valuable alternative to the use of saliva by HIV testing programmes with no medical or nursing staff. Sex Transm Infect. 2012;88(3):218.

42. Spielberg SF, Critchlow HC, Vittinghoff NE, Coletti NA, Sheppard NH, Mayer NK, et al. Home collection for frequent HIV testing: acceptability of oral fluids, dried blood spots and telephone results. AIDS. 2000;14(12):1819-28.

43. Merchant RC, Clark MA, Liu T, Romanoff J, Rosenberger JG, Bauermeister J, et al. Comparison of home-based oral fluid rapid HIV self-testing versus mail-in blood sample collection or medical/community HIV testing by young adult Black, Hispanic, and White MSM: results from a randomized trial. AIDS Behav. 2018;22(1):337-46.

44. Ibitoye M, Frasca T, Giguere R, Carballo-Diéguez A. Home testing past, present and future: lessons learned and implications for HIV home tests. AIDS Behav. 2014;18(5):933-49.

45. Myers EJ, El-Sadr MW, Zerbe MA, Branson MB. Rapid HIV self-testing: long in coming but opportunities beckon. AIDS. 2013;27(11):1687-95.

46. Home Access Health Corporation. HIV-1 Test Service Discontinued [Internet]. Illinois; 2019. https://www.homeaccess.com/hiv-1test-service-discontinued/\#: :text=Through\%2520March\%25203 $1 \% 2 \mathrm{C} \% 25202019 \% 2 \mathrm{C} \% 2520 \mathrm{we}$, use $\% 2520 \mathrm{HIV} \% 2 \mathrm{D} 1 \% 2520 \mathrm{t}$ est $\% 2520$ service.

47. U.S Food \& Drug Administration. OraQuick in-home HIV test [Internet]. Maryland; 2020. https://www.fda.gov/vaccines-blood -biologics/approved-blood-products/oraquick-home-hiv-test.

48. Horvath KJ, Walker T, Mireles L, Bauermeister JA, HightowWeidman L, Stephenson R. A systematic review of technology-assisted HIV testing interventions. Curr HIV/AIDS Rep. 2020;17(4):269-80.

49. Tahlil KM, Ong JJ, Rosenberg NE, Tang W, Conserve DF, Nkengasong S, et al. Verification of HIV self-testing use and results: a global systematic review. AIDS Patient Care STDs. 2020;34(4):147-56.

50. Miller J. COVID-19 is a catalyst for remote sampling and telemedicine [Internet]. Clinical Laboratory News; 2020. https:// www.aacc.org/publications/cln/articles/2020/july/covid-19-is-acatalyst-for-remote-sampling-and-telemedicine.

Publisher's Note Springer Nature remains neutral with regard to jurisdictional claims in published maps and institutional affiliations. 